\title{
Mastering the Art of Scientific Programming or Application Development as a
}

\section{Tool for Scientific Innovation}

\author{
John J. Donovan ${ }^{1}$ \\ ${ }^{1 .}$ CAMCOR, University of Oregon, Eugene, OR, 97403
}

Whether one utilizes a cookbook or "canned" algorithms, the critical distinction is whether the cook or analyst understands the reasoning and purpose behind the various ingredients and steps in the procedure or is just mindlessly following a "recipe". In other words: creative innovation versus mere use.

To be sure, there is a continuum of scientific innovation possible with computer software, including batch files (OS tasks, storage, backup and housekeeping, etc.), scripts (workflow automation, report efficiency, large data sets, etc.), COM interfaces (customized acquisition, data processing automation, etc.) and finally, application development (technique innovation, vertical integration and commercialization).

For example, a spectral interference correction using the method of Gilfrich [1] can be performed post analysis in a spreadsheet, but for many spectral interferences, the matrix correction must be re-calculated subsequent to the interference correction particularly if the interfering concentrations are significant. This is even the case for interferences of trace elements. [2] Therefore by implementing such new correction procedures directly in the matrix iteration code, this interference correction can be performed fully quantitatively.

The rewards for such scientific application development are several, but primary among these are the possibility of implementing of new and useful scientific methods and algorithms for general use as opposed to the traditional route of academic publication, where the possible practical benefits are often not realized as in commercial software. Other less obvious advantages of scientific application development include the elimination of "black boxes"- that is to say, if one wrote it, hopefully one understands it. Or at least one can provide additional "debug" output showing the internal calculations for testing and verification by beta testers and sophisticated users.

Finally, scientific application development, which at times can seem to be dominated by graphical user interface (GUI) and error trapping code, can provide a deep appreciation of the power of scientific models and even more importantly, a greater understanding of the underlying physics involved.

On the other hand, the difficulties and scope of application development can be quite daunting, which is why all such efforts should rely on intimate interaction with the scientific community of users which provides not only real world software testing and also a source of creative ideas, but most importantly, collaboration in these innovation efforts.

Similar development efforts have been pursued by many investigators hoping to improve the accuracy and precision of EPMA throughout the history of micro analysis. [3, 4, 5], but without commercialization the impact of these pioneering contributions were not always fully realized. Commercialization is a difficult bridge to cross for many scientific researchers, though it is possible. 
A few of these "bridges" which must be crossed for successful scientific application development include GUI design (engineering versus scientific considerations, aesthetics, ease of use, etc.), documentation (reference manuals, "how to" guides, frequently asked questions (FAQ), context sensitive help, etc.), support and training issues, application stability and data integrity, and of course a hardware abstraction layer (HAL) in order to support multiple original equipment manufacturer (OEM) instruments each with their own idiosyncrasies.

On this latter point it should be noted that it would be all too easy if all instrument vendors implemented the same orientation and unit conventions. For example Cameca instruments, not surprisingly, utilize a Cartesian coordinate system and micrometer units (um) for their mechanical stages. JEOL instruments on the other hand implement an "anti-Cartesian" coordinate system for their mechanical stages and utilize millimeters $(\mathrm{mm})$ stage units. Of course WDS spectrometer units are also different. Cameca using sin theta units for their spectrometers, while JEOL utilizes so called "L-Value" units for their spectrometers in millimeters.

But once these issues are dealt with using a properly designed HAL, the developer is free to implement innovative methods and algorithms for all instruments in a consistent and integrated fashion.

\section{References:}

[1] JV Gilfrich, LS Birks, JW Criss in "Correction for Line Interferences in Wavelength-Dispersive Xray Analysis", X-Ray Fluorescence Analysis of Environmental Samples, Ed. TG Dzubay. Ann Arbor Science Publ., Ann Arbor, (1978), p. 283.

[2] JJ Donovan, DA Snyder. and ML Rivers (1993) An Improved Interference Correction for Trace Element Analysis, Microbeam Analysis, 2, (1993) 23-28

[3] CE Fiori, CR Swyt, KE Gorlen in "Application of the Top-Hat Digital Filter to a Nonlinear Spectral Unraveling Procedure in Energy-Dispersive X-Ray Microanalysis” Microbeam Analysis (1981), pp. 320-324.

[4] JT Armstrong in "Quantitative Analysis of Silicate and Oxide Materials: Comparison of Monte Carlo, ZAF, and Procedures” Microbeam Analysis (1988), pp. 239-246.

[5] JJ Donovan, ML Rivers, JT Armstrong in "PRSUPR: Automation and Analysis Software for Wavelength Dispersive Electron-Beam Microanalysis on a PC" American Mineralogist 77, (1992) p. 444-445. 